\title{
ANÁLISIS DE LA ESTRUCTURA ESTACIONAL DE LA PRECIPITACIÓN Y SUS CAMBIOS POR TREINTENAS EN LOS ÚLTIMOS 150 AÑOS
}

\author{
José A. LÓPEZ \\ ${ }^{1} A E M E T$. \\ jlopezd@aemet.es
}

\section{RESUMEN}

En los últimos años varios estudios climatológicos han puesto de manifiesto variaciones de la estructura estacional de la precipitación (distribución entre las estaciones del año) en los últimos 50 años aproximadamente. El más importante es un desplazamiento del máximo otoñal típico del clima mediterráneo hacia el centro y suroeste de la Península, lo que sería coherente con el calentamiento global antropogénico.

En esta trabajo se analiza el conjunto de series largas (160 años) de precipitación compiladas y homogeneizadas en AEMET al objeto de extrapolar a ese amplio periodo el estudio de estos cambios de estructura estacional. Además de la descripción se aborda un estudio de la significación estadística de los cambios con técnicas de bootstrap.

Se muestra que a lo largo de las tres treintenas últimas, el desplazamiento entre las dos últimas treintenas de invierno a otoño en el centro y suroeste estuvo precedido por un cambio inverso, esto es, de máximo invernal a otoñal, entre las dos primeras treintenas. Destaca también un descenso importante de la contribución primaveral entre la primera y segunda treintenas de los 150 años estudiados en la región centro y norte.

Palabras clave: precipitación estacional, tendencias en cambios

\begin{abstract}
In the last years several climatological studies have highlighted changes in the seasonal precipitation structure (their distribution) over the last 50 years or so. The main one is the displacement of the autumnal maximum, typical of the Mediterranean climate, towards the center and SW of the Peninsula, which would accord with anthropogenic climate change.

In this work a dataset comprising 66 precipitation series extended over 160 years compiled and homogenized at AEMET is analyzed aiming at carrying over this extended time frame the study of these seasonal changes. In addition to their description an analysis of their statistical significance is included.

It is shown that over the last three 30 -year periods the shift from fall to winter was preceded by an opposite shift from winter to fall between the two 30 -year periods. An outstanding feature is an important fall in the spring part between the two first 30-year periods of the 150 year span.
\end{abstract}


Key words: seasonal precipitation, trends in changes

\section{INTRODUCCIÓN}

A nadie se le oculta la importancia de la pluviometría en un país, como España, con una parte importante del territorio próxima al estrés hídrico, es evidente desde muchos puntos de vista. Probablemente la variable climatológica que más se estudia es la precipitación total anual, por ser la más indicativa de la disponibilidad del recurso hídrico. Pero también tiene un gran interés la distribución de la precipitación a lo largo del año. Pensemos, por ejemplo, en los cultivos de secano y el gran impacto en la cosecha final que puede tener una misma cantidad total de precipitación en el año agrícola repartida entre las cuatro estaciones del año de forma diferente.

En este trabajo la atención se dirige precisamente a cambios en la distribución estacional de precipitaciones que no dependen de la cantidad total de lluvia en el año. Una simplificación importante, en orden a facilitar la visualización y análisis de los resultados, ha consistido en prescindir del verano, es decir, considerar el régimen estacional en un año dado determinado por los valores relativos de precipitación en el otoño, invierno y primavera. Esta simplificación está plenamente justificada para una gran parte del territorio pues la precipitación veraniega es muy pequeña con relación al total anual. Además se ha trabajado exclusivamente con los porcentajes relativos de las tres estaciones: como se explica en el trabajo en detalle, es posible representar un conjunto de tres porcentajes por un punto en el plano, de forma que los tres porcentajes reciban un tratamiento simétrico. Además el paso a porcentajes garantiza una mayor robustez que los totales respectivos frente a posibles rupturas de la homogeneidad de las series. Se ha considerado el año hidrológico de tal forma que el otoño empieza convencionalmente en septiembre.

\section{DATOS}

Los datos utilizados en este estudio provienen de las 66 series largas de precipitación mensual que se formaron y homogeneizaron en AEMET utilizando el programa CLIMATOL desarrollado por J.A. Guijarro. Estas series se extienden a los años 1851-2010, pero aquí se usan los últimos 150 años, 1861-2010.

\section{METODOLOGÍA}

\subsection{EI diagrama triangular}

Matemáticamente los regímenes de precipitación normalizados vienen descritos por tres porcentajes $(o, i, p)$ de otoño, invierno y primavera respectivamente, pero basta para determinarlos con dos números por la condición de suma constante 100 de los porcentajes, de forma que se pueden representar en el plano.

Si representamos en un par de ejes cartesianos los dos números $(o, i)$ el inconveniente es que las líneas de $p$ constantes $(p=100-(o+i))$ están inclinadas $45^{\circ}$ respecto a la horizontal, y por tanto no hay simetría geométrica para los tres porcentajes. El diagrama triangular que se utiliza en este trabajo visualiza la terna $(o, i, p)$ de forma geométricamente simétrica, lo que facilita la interpretación. Supongamos que asignamos al punto de igualdad de porcentajes $(33.3,33.3,33.3)$ el origen del plano O. 
Para representar cualquier otro punto $(o, i, p)$ consideremos la diferencia dif $=(o-33.3$, $i-33.3, p$-33.3) que vale $(0,0,0)$ para el punto $\mathrm{O}$. Es fácil ver que la suma de las tres componentes de dif es siempre igual a 0 y que podemos representarlo en el plano de la siguiente forma: trazamos desde $\mathrm{O}$ tres vectores base de longitud $100-33.3=66.7$ separados ángulos de $120^{\circ}$ (de forma que son simétricos respecto a O). Se puede probar que para cada punto del plano la suma de sus tres proyecciones (con signo) sobre estos vectores base es nula dada la simetría respecto a O. Podemos por tanto representar a dif por el punto $\mathrm{P}$ tal que su proyección sobre cada uno de los tres vectores es igual respectivamente a cada componente de dif.

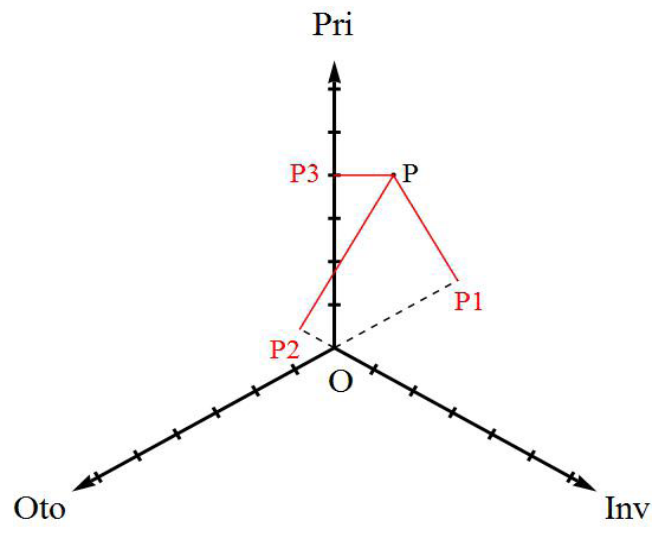

Fig.1: Explicación del diagrama triangular (ver texto)

Esto se aclara con un ejemplo numérico. En el diagrama (fig. 1) se representan los tres vectores básicos asociados a las tres estaciones. Cada marca sobre los vectores representa $10 \%$ de porcentaje. Para ver los porcentajes de un punto cualquiera como $\mathrm{P}$ hay que primeramente hallar las componentes de su vector dif, esto es, las proyecciones ortogonales sobre cada vector estacional, marcadas respectivamente con P1, P2 y P3 en el gráfico. En este caso valen $(-31,-9.7,40.0)$, y por tanto para hallar los porcentajes finales para $\mathrm{P}$ sumamos el valor correspondiente al origen $\mathrm{O}$ (33.3, $33.3,33.3)$ y obtenemos finalmente $(2.1,24.6,73.3)$. De este procedimiento se deduce que en el diagrama triangular los extremos de los vectores básicos representan los puntos de porcentaje 100 para cada una de las estaciones, y que a lo largo de los lados del triángulo cuyos vértices son esos extremos se alinean los puntos con porcentaje nulo en alguna estación, en concreto aquella cuyo vector es perpendicular al lado. Así, como $\mathrm{P}$ está próximo al lado del triángulo exterior perpendicular a otoño tiene muy poco porcentaje esa estación, mientras que la relativa proximidad al extremo Pri indica porcentaje alto en primavera.

\subsection{Significación estadística de los cambios de régimen pluviométrico normalizado}

Para analizar la significación estadística de los cambios en los totales de precipitación medios de cada estación del año entre dos periodos sucesivos de 30 años se ha aplicado una técnica de "bootstrap" (López Díaz, 2015). El problema se plantea de la siguiente forma: en cada uno de las dos treintenas tenemos un vector de 3 componen- 
tes con los totales estacionales, que se normalizan en forma de porcentajes de forma que sumen 100. Para juzgar si el segundo vector, correspondiente a la segunda treintena, supone un cambio significativo estadísticamente respecto al primero, es necesario estimar la distribución del primer vector de porcentajes bajo las condiciones climáticas de la primera treintena. Una forma de aproximar estas condiciones climáticas sin introducir hipótesis ad hoc consiste en utilizar muestras aleatorias de esos 30 años que simulen muestras provenientes de ese tipo de régimen pluviométrico. Además se precisa de una "métrica" que mida en escala ordinal la diferencia entre dos de esos vectores de porcentajes y los ordene según su distancia a uno determinado (esto es necesario para calcular un p-valor) En concreto se han seguido los siguientes pasos:

A. La métrica elegida para valorar la desemejanza entre dos vectores de totales estacionales normalizados es la euclídea (raíz de suma de cuadrados del vector diferencia)

B. Se forman los vectores de totales estacionales observados normalizados en las dos treintenas a comparar y se calcula su distancia euclídea

C. Se generan muestras por bootstrap de vectores normalizados para la primera treintena (como proviniendo del mismo régimen). Para ello se muestrean aleatoriamente los años por bloques de longitud media 5 años por la técnica de bootstrap geométrico (para asegurar estacionariedad)

D. Para cada vector aleatorio generado se halla la distancia al vector observado con la métrica de A.

E. Con la población de distancias (10.000) generadas se valora la significación estadística de la distancia observada hallando la probabilidad de tener una distancia mayor.

\section{DESCRIPCIÓN DE RÉGIMEN MEDIO Y CAMBIOS ENTRE TREINTENAS}

Se ha dividido el estudio abordado de los cambios de los regímenes estacionales de precipitación (sin verano) en dos periodos de forma en parte convencional. El primero abarca los últimos 90 años, 1921-2010, en los cuales puede aceptarse que la densidad de observatorios es suficiente para conseguir una descripción fiable, mientras que el segundo incluiría también los anteriores 60 años, 1861-2010, en que la fiabilidad de los resultados obtenidos disminuye. Se comienza analizando para el periodo completo el régimen estacional medio.

\subsection{Régimen estacional medio en 1861-2010}

En la fig. 2 se presentan para 28 observatorios los diagramas triangulares con los valores medios de los porcentajes de precipitación medios en oto-inv-prim. En cada diagrama los ejes corresponden a $10 \%$ y el origen representa el punto de simetría de porcentajes. Como se delinea en la figura se distinguen con claridad dos grandes zonas según la distribución estacional: la mediterránea costera con predominio otoñal y la atlántica con predominio invernal. Se distingue también una zona mediterránea interior con predominio simétrico primavera-otoñal. Por último hay una cuña invertida desde el centro de la Península con apertura hacia el norte sin dominancia estacional clara. 


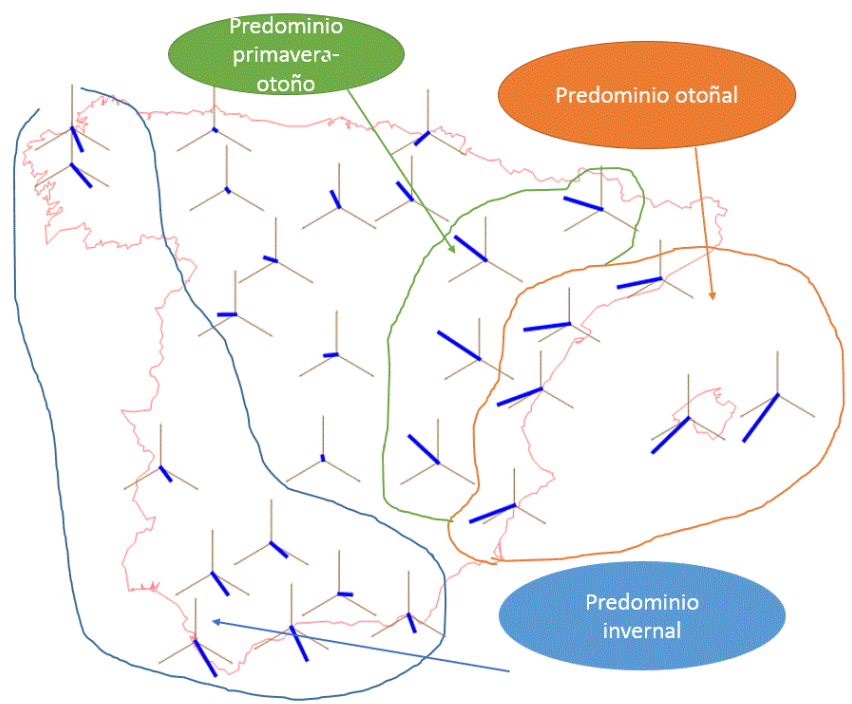

Fig. 2: Diagramas triangulares de \% de precipitación en las tres estaciones del año oto-invprim. El origen de cada diagrama es el punto de igualdad de $\%$ y la escala de los ejes $=10 \%$.

\subsection{Cambios en 1921-2010}

Los porcentajes de los totales estacionales de las tres treintenas sucesivos que cubren este periodo de 90 años se han representado mediante diagramas triangulares en el mapa de la fig. 3 ((López Díaz, 2016). En cada diagrama la línea roja une los puntos correspondientes a las dos primeras treintenas (1921-1950, 1951-1980), y la línea azul une los puntos entre las dos últimas treintenas (1951-1980, 1981-2010). Los vectores básicos miden en estos diagramas 4\%, lo que da idea de que los cambios de porcentajes medios entre treintenas son pequeños, del orden del 4\%. El origen de cada diagrama representa los porcentajes estacionales medios para todo el periodo de 90 años para cada observatorio, al objeto de que el diagrama de cada observatorio ocupe un espacio reducido.

En el mapa observamos en los observatorios de la vertiente atlántica un patrón de $\mathrm{V}$ tendida aproximadamente en horizontal y con el vértice a la derecha, con el tramo rojo en la parte superior de la V. Esto indica, de acuerdo a la interpretación de 3.1, que entre las dos primeras treintenas hay un aumento marcado de la precipitación invernal $\mathrm{y}$ un descenso de la precipitación primaveral, mientras que el cambio entre las dos últimas treintenas implica un aumento acentuado de la lluvia otoñal y otro descenso de la primaveral. Cuando cruzamos una línea norte-sur imaginaria entre Bilbao y Almería, que correspondería al paso a la vertiente mediterránea, el cambio en los diagramas es muy claro. En primer lugar la forma de los mismos es más variable, y la magnitud de los cambios en general inferior a la vertiente atlántica. En la cuenca del Ebro vemos patrones de cambio similares a los de la vertiente atlántica en la misma latitud. En cambio en las estaciones costeras mediterráneas y las Baleares los cambios son de escasa magnitud. 


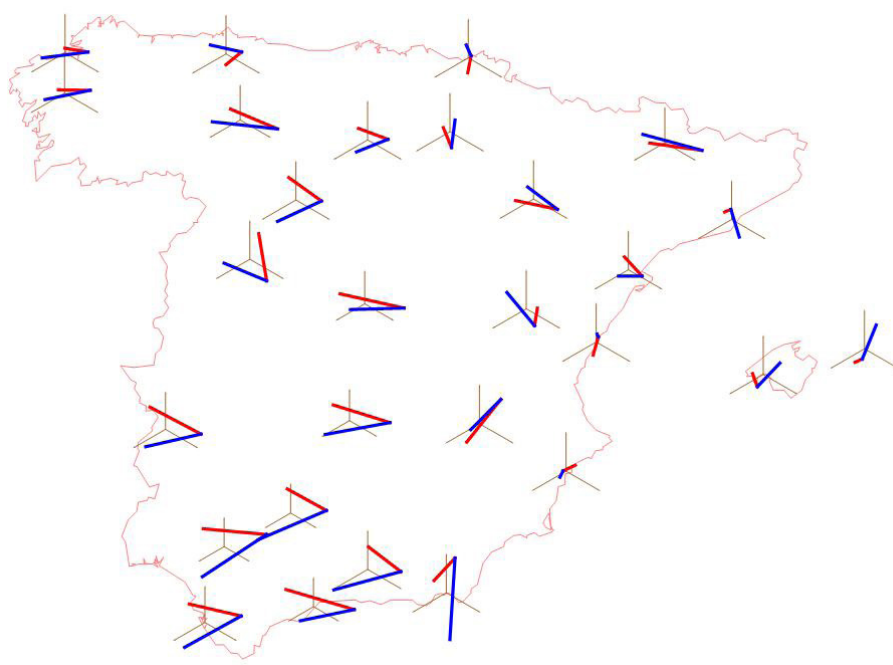

Fig.3: Mapa con los diagramas triangulares para los porcentajes medios estacionales en las tres treintenas de 1921-2010

La magnitud de los cambios es máxima en el cuadrante SO y mínima a lo largo de la costa mediterránea.

\subsection{Cambios en 1861-2010}

En cada diagrama triangular de la fig. 4 los 5 puntos de cada treintena están unidos por rectas negras, salvo la roja que une las dos primeros treintenas, y la verde une las dos últimas treintenas. Como en la fig. 2 la escala de los ejes del diagrama es 4\% el origen es el punto medio. Se ha añadido un punto azul en cada diagrama que representa esos porcentajes estacionales medios a lo largo de todo el periodo de 150 años, con escala de los ejes $10 \%$ referidos al punto de simetría de porcentajes.

Quizá el rasgo más llamativo que se aprecia en estos diagramas es que el patrón en zigzag oto-inv-oto de los últimos 90 años en la vertiente atlántica descrito en 3.2, que en los diagramas corresponde al segmento verde y el adyacente, no es el único patrón importante en términos de amplitud y prevalencia espacial. En el periodo 1861-1920 (segmento rojo y adyacente) se aprecia una clara disminución del porcentaje primaveral en la vertiente atlántica y parte de la cuenca del Ebro, de magnitud comparable a la del patón en zigzag consecutivo.

En el cuadrante SO puede distinguirse un patrón regular a lo largo de todo el periodo, de forma particularmente nítida en San Fernando (Cádiz). Cabe descomponerlo en dos componentes:

1) una componente oscilatoria, de mayor amplitud, consistente en un patrón en zigzag otoño-invierno otoño, con una amplitud total que en San Fernando alcanza aproximadamente un $8 \%$, y con un periodo temporal de aproximadamente toda la longitud de la serie, unos 150 años. Por tanto en estos observatorios vemos que el patrón en zigzag de los últimos 90 años se prolonga hacia atrás hasta extenderse a los 150 años. 


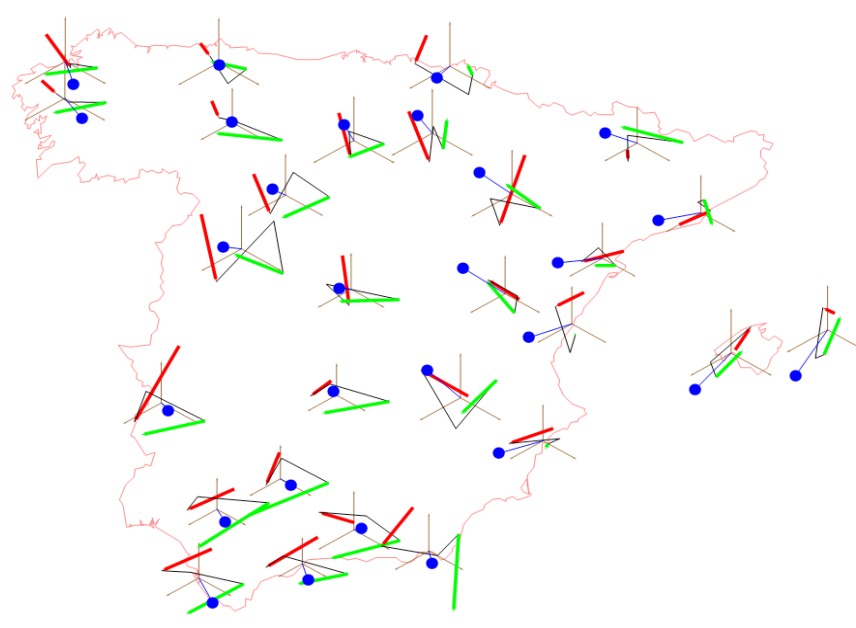

Fig. 4: Diagramas triangulares para los \% oto-inv-prim medios de los 5 treintenios en 18612010.

2) una segunda componente definida por un descenso a ritmo prácticamente constante del porcentaje primaveral que así mismo cubre todo el periodo, con un ritmo aproximado de $6 \% / 100$ años.

Los cambios en la vertiente mediterránea, en su franja costera, son significativamente menores en magnitud, y en varios casos como Baleares o Tarragona, muestran un curioso patrón en ciclo cerrado a lo largo de estos 150 años.

\subsection{Síntesis de los cambios estacionales en los últimos 150 años.}

En la fig. 5 se han dibujado los 4 diagramas triangulares correspondientes a las evoluciones estacionales promedio por treintenas promediados para cada grupo semejante de observatorios según régimen estacional medio descritos en el apartado 3.1 (fig. 2). A cada grupo se le ha asignado el nombre abreviado de un observatorio característico representante del grupo: ZAR para el grupo mediterráneo interior, MAD para grupo centro y norte, BAR para el grupo mediterráneo costero y SFER para el grupo atlántico.

En la fig. 5 todos los puntos del diagrama tienen el mismo origen de coordenadas que es el punto de simetría de porcentajes. Las marcas de los ejes corresponden a $2 \%$. De esta forma podemos apreciar fácilmente tanto los rasgos de la evolución inter-treintena dentro de cada grupo (segmentos) como la situación del grupo respecto a los demás en términos de su régimen estacional promedio (que determina el origen de su diagrama).

Se aprecia claramente que la magnitud de las variaciones inter-treintenas, dadas por el tamaño de los segmentos, es por orden decreciente SFER, MAD, ZAR y BAR, y por tanto aumenta en sentido NE-SO. Vemos la separación clara entre los centros de los grupos comparado con los cambios inter-treintenas dentro de cada grupo (lo que confirma la buena composición de los grupos), aunque los grupos centro-norte MAD y atlántico SFER están bastante próximos. 


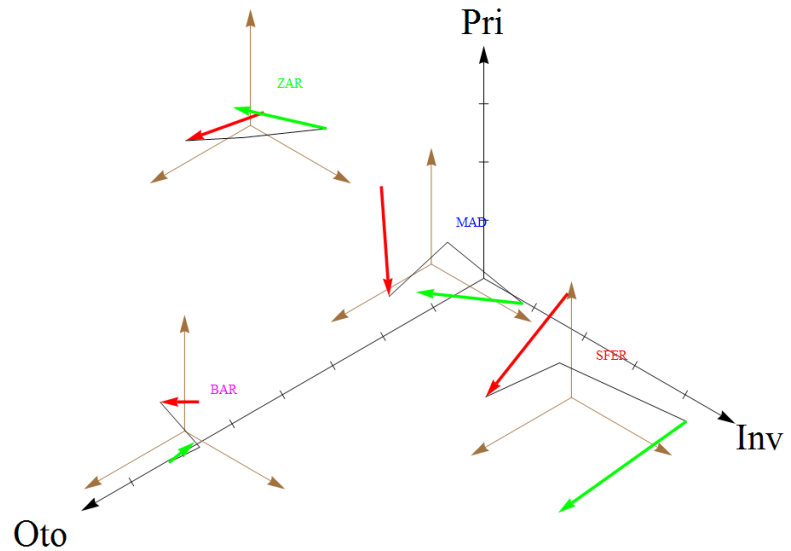

Fig. 5: Diagramas triangulares para la evolución por treintenas de los cuatro grupos de observatorios según su régimen estacional medio

\section{SIGNIFICACIÓN ESTADÍSTICA DE LOS CAMBIOS}

\subsection{Periodo 1921-2010: distribución espacial}
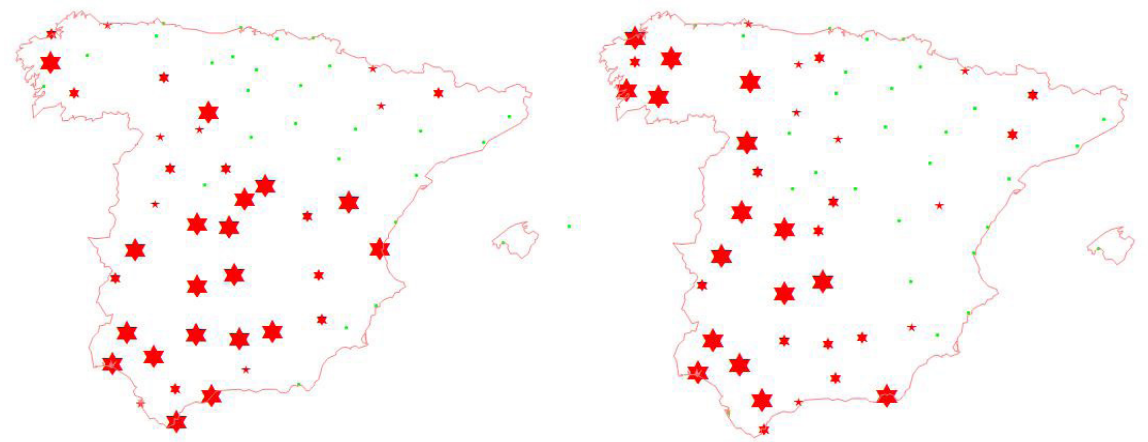

Fig. 6: Cambios significativos en los porcentajes de precipitación estacionales entre las treintenas 1921-1950/1951-1980 (izq) y 1951-1980/1981-2010 (der). El tamaño de las estrellas rojas indica el p-valor, 0.01, 0.05, 0.10 respectivamente decreciendo en tamaño.

Puntos verdes: observatorios sin cambio significativo.

Para los últimos 90 años analizados en este trabajo se ha aplicado la metodología esbozada en 3.2 con objeto de comparar los grados de significación estadística para los cambios entre las dos primeras treintenas del periodo con los cambios entre las dos últimas. Como se vio en 4.2 en el centro y oeste de la Península esos cambios forman el patrón en $\mathrm{V}$ tendida hacia la izquierda de la fig. 3, es decir, oscilación otoño $\rightarrow$ invierno $\rightarrow$ otoño.

En la fig. 6 se aprecia que los cambios significativos entre las treintenas 19211950/ 1951-1980 (izq.) y 1951-1980/1981-2010 (der.) muestran una distribución es- 
pacial bastante similar. Se concentran fundamentalmente hacia el SO de una diagonal entre Asturias y Murcia.

Es claro que las zonas con cambios significativos se solapan en los dos mapas de la fig. 6, lo que es indicativo de que es la treintena central común a ambos, 1951-1980, la que tiene en estos 90 años un carácter atípico, de modo que tanto el cambio de la primera treintena a esta central, como de la central a la última, son altamente significativos.

\subsection{Análisis sumario para el periodo 1861-2010}

Para tratar de confirmar de forma objetiva esta conjetura se ha procedido a aplicar la técnica de este apartado a todas las 10 parejas de treintenas formadas por las 5 treintenas que cubren el periodo 1861-2010. Con estos resultados se puede definir un índice de cambios para cada treintena $\mathrm{T}$ del siguiente modo: se suman todos los observatorios con cambio significativo al 5\%/1\% para todas las parejas de treintenas en que interviene la treintena $\mathrm{T}$ ( 4 en total, la 2 por ejemplo interviene en los pares 1-2, 2-3, 2-4 y 2-5). Este número se normaliza dividiéndolo por su máximo teórico, igual a 4 x 66 observatorios.

\begin{tabular}{|c|c|c|c|c|c|c|}
\hline \multicolumn{2}{|c|}{ Treintena } & $1861-1990$ & $1891-1920$ & $1921-1950$ & $1951-1980$ & $1981-2010$ \\
\hline \multirow{2}{*}{$\begin{array}{c}\text { Índice } \\
\text { Camb. }\end{array}$} & $1 \%$ & $56.1 \%$ & $36.4 \%$ & $32.2 \%$ & $45.1 \%$ & $34.1 \%$ \\
\cline { 2 - 7 } & $5 \%$ & $71.2 \%$ & $52.7 \%$ & $46.6 \%$ & $61.7 \%$ & $48.1 \%$ \\
\hline
\end{tabular}

Tabla 1: Índice relativo de cambios respecto a otras treintenas para cada treintena, a los niveles de significación 1\% y 5\%.

En la tabla 1 se reflejan los valores de este índice relativo de cambios al $1 \% \mathrm{y}$ al 5\%; como se puede observar, efectivamente en los últimos 90 años la treintena 1951-1980 destaca con claridad sobre las otras dos adyacentes temporalmente. Pero puede verse que, en realidad, sería la primera treintena 1861-1990 la más separada del resto, incluso con un porcentaje medio de cambios con relación a las otras cuatro de más del 50\% al nivel de significación 1\%. El sentido dominante de los cambios que marcaron esa treintena se infiere de la fig. 4 (o más sintéticamente de la fig.5), es decir, en las zonas vertiente atlántica y centro hubo una disminución acusada del porcentaje estacional primaveral (segmentos rojos de esas figuras). Naturalmente es necesario tomar estos resultados con cautela por la fiabilidad que podamos atribuir a los datos sobre una red de 66 observatorios en ese lejano periodo.

\section{RESULTADOS Y DISCUSIÓN}

Se describen los cambios en los regímenes de precipitación estacional (sin verano) en España peninsular sobre la base de periodos de 30 años consecutivos a lo largo de los últimos 150 años, 1861-2010. El aspecto descriptivo se ha basado en gran medida en un diagrama triangular que permite visualizar de forma sintética los cambios. Se muestran también los resultados de un análisis de significación estadística de los cambios de régimen estacional de precipitación entre treintenas. 
Estos análisis concuerdan con estudios previos (González-Hidalgo, 2009 y 2013, Martin de Luis, 2010) que han puesto de manifiesto que ha existido en los últimos 60 años aproximadamente un marcado desplazamiento de la contribución invernal a la otoñal en la vertiente atlántica y zona centro.

La más dilatada escala temporal que aporta este trabajo permite poner esta importante oscilación en perspectiva. En concreto queda patente que esa importante basculación invierno $\rightarrow$ otoño vino precedida en aproximadamente las mismas áreas por otra de sentido opuesto otoño $\rightarrow$ invierno. De esto cabe concluir que de las tres últimas treintenas ha sido la intermedia 1951-1980 la de carácter climático en este aspecto más disonante.

Extendiéndose a todas las cinco treintenas de este estudio resulta que la más disonante de todas, en términos de una medida objetiva de disparidad, fue en realidad la primera 1861-1990. Su carácter atípico consistió en un marcado descenso primaveral en áreas similares a las afectadas por la oscilación otoño $\rightarrow$ invierno $\rightarrow$ otoño de los últimos 90 años. En cambio la vertiente mediterránea muestra cambios menores en conjunto sin patrones de dominancia comparable a los que afectan a la atlántica-centro.

Cabe concluir que la variabilidad de la precipitación requiere de periodos de muchos años para una caracterización satisfactoria en la Península, al menos en la vertiente atlántica.

\section{REFERENCIAS}

González-Hidalgo, J.C. et al. (2009): Monthly precipitation trends on the Mediterranean fringe of the Iberian Peninsula during the second-half of the twentieth century (1951-2000) DOI: 10.1002/joc.1780 International Journal of Climatology Volume 29, Issue 10, pages 1415-1429

González-hidalgo, J.C. et al (2013): Las tendencias de las precipitaciones en España en el período 1945-2005 En "Fenómenos meteorológicos adversos en España", C. García-Legaz y F. Valero eds.

López Díaz, J.A. (2015): Changes in seasonal precipitation regimes in Spain: a statistical significance study. Presentado en "International Symposium CLIMATEES 2015", Tarragona 2015.

López Díaz, J.A. (2016): Cambios en los regímenes pluviométricos estacionales en España “Calendario Meteorológico 2016”, AEMET, ISSN-0213 3849, pags. 283 287

Martin de Luis et al. (2010): Changes in seasonal precipitation in the Iberian Peninsula during 1946. Global and Planetary Change 74 (2010) 27-33 